\title{
COBINING ABILITY IN RELATION TO GENETIC DIVERSITY IN COTTON (G. BARBADDENSE L.)
}

\author{
Shaker S.A., A.E.I. Darwesh, M.E. Abd El-Salam \\ Cotton Research Institute, Agricultural Research Center, Giza, \\ Egypt
}

\begin{abstract}
The experiments were carried out at Sakha Agriculture Research Station, Cotton Research Institute, Agriculture Research Center, Egypt. The aim of this investigation was to study heterosis and combining ability for the most important characters of cotton (seed cotton yield, lint yield, boll weight, seed index, lint percentage, lint index and hallo length). Ten cotton genotypes TNB, Karsheneski 2 , G.45, G.89 x G.86, G.86, G.94, G. 93, CB.58, G.75 x Sea and Australian $_{12}$ were crossed in half diallel. These parents and their respective $45 F_{1}$ crosses were evaluated in a randomized complete blocks design. The results obtained could be summarized as follows:-

Highly significant mean square values were obtained for genotypes, general combining ability and specific combining ability for all the studied characters. The best general combiner for all studied traits were parents G.94 and $\mathrm{G} .75 \times$ sea. Also the best combination for most of studied characters were crosses G.45 x CB 58, TNB x Aus. ${ }_{12}$, Kar. . $_{2}$ ( G.94, G45 x CB 58, G.94 x (G.75 x sea) and G.93 x (G.75 x sea).

The first three principle components were significant and accounted about $90.3 \%$ of the total variability of all characters. Which having maximum Eigen value. Lint index followed by lint percentage, boll weight and lint yield were a primary source of variation in the first ax. The second ax was principally affected by seed cotton yield and hallo length. The ten parents in this study were grouped in different clusters and there were accordance between parental diversity and significant general and specific combining ability.
\end{abstract}

Key words: Combining ability, principle components diversity, and cotton.

\section{INTRODUCTION}

The choice of selection breeding procedures for genetic improvement of cotton or any crop, is largely conditioned by the type and relative amounts of genetic variance components in the population. The exploitation of genetically diverse stock in cross combinations helps to identify promising hybrid and / or to develop superior inbred lines. The diallel cross analysis has been used by many investigators to assist in the investigation of nature of heterosis and partition in the genetic variance. Many investigators studies general and specific combining ability and type of gene action using 
diallel mating design (Khan et al. (2011), Amein et al. (2013), Abou El-Yazied et al. (2014) and Abdel-Hafez et al. (2016) and found significant general and specific combining ability for yield and its contributed characters which reflect the importance of additive and non-additive gene effects in the inheritance of such characters.

Estimation of genetic diversity is an important step for any breeding program, but not the last one. Another helpful issue to be evaluated is the relative importance of the characters. Though plant breeders, often measure several characters simultaneously in cotton development, then it is possible to estimate the genetic divergence using multivariate method exist.

Multivariate technique could resolve several phenotypic measurements into fewer, more interpretable and more easily visualized dimensions such an analysis which use principal components (Hair et al. 1987), seemed to elucidate pattern of variation in agronomic attributes and to obtain the initial factor solution using Eigen values. These values measure the explained variance associated with each variable and refer to its contribution to the whole divergence. Principle component analysis (PCA) reflects the importance of the largest contributor to the total variation at each ax for differentiation Sharma, 1998. This analysis seemed to elucidate patterns of variation in agronomic attributes which are of economic importance and obtain entail factor solution using, Eigen values. These values could measure the explained variance associated with each vector, variable. The efficacy of the genetic divergence as a criterion for choosing parents for crossing programmers has been reported by several workers (Sandhu and Boparai, 1997; Patial et al., 1999; ElMansy, 2005; Gooda, 2007 and Abou El- Yazied et al., 2009). Moreover, principal component analysis and factor analysis have analogous efficacy to determine the most suitable combinations and grouping the varied genotypes into varied groups. Seyam et al., 1984 used factor analysis in determining characters that could be selected for high yield. Abd El- Sayyed et al., 2000 and El- Mansy et al., 2008 used principal component and cluster analysis to create genetic variability in Egyptian cotton.

Thus information about genetic variance of parents, GCA, and SCA will be helpful for the necessary testing of parents and crosses before their use in breeding cultivars suitable in future. These studies indicate the necessity to develop cotton cultivars with high stability for agronomic performance in planting growing system.

Therefore, the main objectives of the present study are to study the behavior of genotypes, general and specific combining ability and to select the suitable parents and combinations. Also, to determine genetic diversity among parental cotton genotypes as well as $F_{1}$ 
hybrids combinations by using multivariate analysis to select the most suitable combinations and parents.

\section{MATERIALS AND METHODS}

The present investigation was carried out at Sakha Agriculture Research Station, Cotton Research Institute, Agriculture Research Center, Egypt, during the two growing seasons of 2015 and 2016. Ten parents genotypes of wide divergent origin namely TNB $\left(P_{1}\right)$, Karsheneski $i_{2}\left(\right.$ Kar. $\left._{2}\right)\left(P_{2}\right)$, Giza 45 (G. 45) (P3), Giza $89 \times$ Giza 86 (G. $89 \times$ G. 86) $\left(P_{4}\right)$, Giza 86 (G. 86) ( $\left.P_{5}\right)$, Giza 94 (G. 94) $\left(P_{6}\right)$, Giza 93 (G. 93) $\left(P_{7}\right)$, CB.58 $\left(P_{8}\right)$, Giza $75 \times$ Sea (G. $75 \times$ Sea) $\left(P_{9}\right)$ and Australian $_{12}$ (Aus. $\left.{ }_{12}\right)\left(P_{10}\right)$ were crossed in a half diallel mating design to produce $45 \mathrm{~F}_{1}$ hybrids in 2015 season. The $45 \mathrm{~F}_{1}$ hybrids and their parents were grown in 2016 season in a randomized complete blocks design with three replications was used. Plot size was one row, $5 \mathrm{~m}$ long and $0.7 \mathrm{~m}$ wide with $0.3 \mathrm{~m}$. hill spacing. Hills were thinned to two plants per hill. The normal cultural practices for cotton production were performed at proper time. Data were recorded on ten individual guarded plants chosen at random from each plot in middle ridge for $F_{1}$ and their parents.

Collected data: The collected data were recorded for the following characters: Seed cotton yield (SCY) / plant (g), Lint yield (LY) / plant (g), Boll weight (BW) ( g ), Seed index (SI) ( g ), Lint percentage (LP) (\%), Lint index (LI) (g), and Fiber length (hallo length) ( $\mathrm{HL})(\mathrm{mm})$.

\section{Statistical procedure:}

Data were subjected to method of statistical analysis. Firstly, analysis of variance was done as outlined by Snedecor and Cochran (1982), general combining ability (GCA) and specific combining ability variance (SCA), effects of the hybrids were estimated according to Griffing, s (1956). Also, heterosis over better parent was calculated as the percentage of increase better parent (BP) of each cross as follow: Heterosis relative to better parent (B.P) \% $=\left(\left(F_{1}-B P\right) / B P\right) \times 100$.

After this step, multivariate technique was conducted by using principal component analysis according to Haire et al., (1987) this analysis was calculated from a matrix based on correlation between the studied characters for all genotypes. The genotypes were also grouped as diagram on principal components axes. All this computations were performed using SPSS Computer procedures. 
RESULTS AND DISCUSSION

The results in Table (1) exhibited that the mean squares of genotypes, parents and crosses were highly significant for all characters except for parent versus crosses that was insignificant for boll weight, lint index and hallo length. Also, the mean squares of general combining ability and specific combining ability were highly significant for all characters. The previous results indicated that the experimental materials possessed considerable amount of variability and the two types of combining ability were involved in the genetic expression of these characters. Higher proportion of variance for general combining ability than specific combining ability, suggested that the major roll of additive and non-additive gene effects in the genetic control of these characters with the predominance of additive ones.

These results support the findings of Ahuja and Tuteja (2000), Tuteia et al. (2003), El- Lawendy et al. (2008) and Abdel-Hafez et al. (2016).

Table 1. Mean squares of parents and F1 for all studied characters

\begin{tabular}{|c|c|c|c|c|c|c|c|c|}
\hline S O V & df & SCY/P & LCY/P & BW/g & SI/g & LP $(\%)$ & LI/g & HL/cm \\
\hline Replications & 2 & 9.41 & 3.05 & 0.12 & 0.001 & 1.669 & 0.157 & 0.065 \\
\hline Genotypes & $(54)$ & $681.3^{\star \star}$ & $123.91^{\star \star}$ & $0.328^{\star \star}$ & $1.76^{\star \star}$ & $18.015^{\star \star}$ & $3.131^{\star \star}$ & $5.137^{\star \star}$ \\
\hline Parents & 9 & $721.04^{\star \star}$ & $182.45^{\star \star}$ & $0.5535^{\star \star}$ & $2.4852^{\star \star}$ & $33.779^{\star \star}$ & $6.1743^{\star \star}$ & $5.9824^{\star \star}$ \\
\hline Crosses & 44 & $683.25^{\star \star}$ & $113.88^{\star \star}$ & $0.2881^{\star \star}$ & $1.5997^{\star \star}$ & $14.979^{\star \star}$ & $2.5771^{\star \star}$ & $5.0678^{\star \star}$ \\
\hline P. V. Cross & 1 & $237.76^{\star \star}$ & $38.11^{\star \star}$ & 0.07316 & $2.2638^{\star \star}$ & $9.719^{\star \star}$ & 0.1069 & 0.5798 \\
\hline GCA & 9 & $1025.62^{\star \star}$ & $280.20^{\star \star}$ & $0.9217^{\star \star}$ & $4.364^{\star \star}$ & $75.888^{\star \star}$ & $12.6053^{\star \star}$ & $4.6717^{\star \star}$ \\
\hline SCA & 45 & $612.43^{\star \star}$ & $92.65^{\star \star}$ & $0.2097^{\star \star}$ & $1.2387^{\star \star}$ & $6.4397^{\star \star}$ & $1.2359^{\star \star}$ & $5.2302^{\star \star}$ \\
\hline error & 108 & 31.37 & 4.91 & 0.091 & 0.264 & 0.448 & 0.129 & 0.638 \\
\hline
\end{tabular}

${ }^{* \star}$ and * Significant at 0.05 and 0.01 levels of probability respectively.

\section{Mean performance}

Significant of differences among genotypes exhibited that there are difference between these materials. The mean performance for parents and all crosses are presented in Table (2). The results indicated that the highest parent was Giza $75 \times$ Sea $\left(P_{9}\right)$ for seed cotton yield/plant, lint yield / plant, boll weight, seed index, lint percentage and lint index with the mean values of (98.96 g., $41.33 \mathrm{~g}$., 3.90 g., 12.03 g., $41.80 \%$ and 8.64 g. respectively). 
Table 2: Mean performance of parents and F1 for all studied characters

\begin{tabular}{|c|c|c|c|c|c|c|c|c|}
\hline Genotypes & $\begin{array}{c}\text { No. of } \\
\text { genotype }\end{array}$ & $\begin{array}{c}\text { SCY/Pla } \\
\text { nt }\end{array}$ & $\begin{array}{c}\mathrm{LCY} / \mathrm{PI} \\
\text { ant }\end{array}$ & BW, $\mathbf{g}$ & $\begin{array}{r}\text { SI, } \\
\text { g }\end{array}$ & $\begin{array}{c}\mathrm{LP}, \\
\%\end{array}$ & $\begin{array}{l}\text { LI, } \\
\text { g }\end{array}$ & $\begin{array}{l}\mathrm{HL}, \\
\mathrm{mm}\end{array}$ \\
\hline TNBXKar. 2 & 1 & 51.93 & 17.28 & 2.74 & 9.77 & 33.25 & 4.87 & 37.83 \\
\hline TNBXG .45 & 2 & 49.61 & 16.72 & 3.02 & 10.47 & 33.69 & 5.32 & 39.83 \\
\hline TNBX(G. 89XG. 86) & 3 & 54.15 & 20.28 & 3.26 & 10.35 & 37.42 & 6.19 & 38.67 \\
\hline TNBXG. 86 & 4 & 62.66 & 24.07 & 3.45 & 10.33 & 38.39 & 6.45 & 39.67 \\
\hline TNBXG. 94 & 5 & 72.39 & 25.26 & 3.91 & 11.71 & 34.86 & 6.26 & 40.33 \\
\hline TNBxG. 93 & 6 & 70.81 & 24.66 & 3.49 & 10.31 & 34.81 & 5.51 & 40.00 \\
\hline TNBxCB 58 & 7 & 42.64 & 15.60 & 3.03 & 9.73 & 36.64 & 5.63 & 36.50 \\
\hline TNBxG. 75XSea & 8 & 64.80 & 24.80 & 3.40 & 10.45 & 38.25 & 6.47 & 38.67 \\
\hline TNBXAus. 12 & 9 & 83.59 & 30.33 & 3.21 & 9.33 & 36.30 & 5.32 & 37.00 \\
\hline Kar. 2XG. 45 & 10 & 30.85 & 10.39 & 2.73 & 9.73 & 33.60 & 4.93 & 39.17 \\
\hline Kar. 2X(G. 89XG. 86) & 11 & 79.10 & 27.70 & 3.11 & 9.82 & 35.02 & 5.29 & 38.67 \\
\hline Kar. 2XG. 86 & 12 & 70.82 & 26.01 & 2.83 & 10.06 & 36.72 & 5.84 & 38.50 \\
\hline Kar. 2XG. 94) & 13 & 66.62 & 24.61 & 3.33 & 10.99 & 36.93 & 6.44 & 39.67 \\
\hline Kar. 2XG. 93 & 14 & 71.04 & 23.73 & 2.97 & 9.74 & 33.39 & 4.89 & 41.83 \\
\hline Kar. 2XCB 58 & 15 & 63.18 & 21.34 & 2.83 & 9.74 & 33.81 & 4.98 & 39.67 \\
\hline Kar. 2X(G. 75XSea) & 16 & 43.30 & 16.50 & 3.31 & 10.29 & 38.09 & 6.33 & 39.17 \\
\hline Kar. 2XAus. 12 & 17 & 40.13 & 14.32 & 3.02 & 9.61 & 35.70 & 5.34 & 37.50 \\
\hline G. 45X(G. 89XG. 86) & 18 & 63.34 & 25.32 & 2.78 & 10.24 & 40.00 & 6.83 & 36.33 \\
\hline G. $45 X G .86$ & 19 & 68.30 & 27.21 & 3.58 & 10.50 & 39.82 & 6.94 & 39.33 \\
\hline G. 45XG. 94 & 20 & 45.71 & 16.67 & 2.95 & 9.68 & 36.47 & 5.56 & 39.50 \\
\hline G. 45XG. 93 & 21 & 48.16 & 18.54 & 3.27 & 10.26 & 38.48 & 6.42 & 36.33 \\
\hline G. 45XCB 58 & 22 & 95.37 & 37.34 & 3.41 & 10.79 & 39.14 & 6.95 & 38.67 \\
\hline G. 45X(G. 75XSea) & 23 & 94.95 & 35.74 & 2.96 & 9.22 & 37.64 & 5.56 & 39.83 \\
\hline G. 45XAus 12 & 24 & 57.34 & 21.39 & 3.11 & 9.72 & 37.31 & 5.79 & 38.50 \\
\hline (G. 89XG. 86)XG86 & 25 & 69.34 & 27.46 & 2.85 & 10.72 & 39.58 & 7.02 & 39.50 \\
\hline (G. 89 XG. 86)X G. 94 & 26 & 57.97 & 23.14 & 2.86 & 10.46 & 39.91 & 6.94 & 37.83 \\
\hline (G. 89XG. 86)XG. 93 & 27 & 61.81 & 23.12 & 3.25 & 10.76 & 37.43 & 6.45 & 40.00 \\
\hline (G. 89XG. 86)XCB 58 & 28 & 61.17 & 22.69 & 3.29 & 9.45 & 37.11 & 5.58 & 39.00 \\
\hline (G. 89XG. 86)XG. 75XSea & 29 & 64.69 & 25.28 & 3.66 & 10.92 & 39.09 & 7.01 & 39.33 \\
\hline (G. 89XG. 86)xAus12 & 30 & 61.09 & 23.23 & 3.23 & 10.76 & 38.03 & 6.61 & 36.83 \\
\hline G. 86 X G. 94 & 31 & 54.98 & 22.64 & 3.45 & 11.07 & 41.22 & 7.76 & 37.17 \\
\hline G. 86 XG. 93 & 32 & 82.60 & 31.05 & 3.00 & 9.74 & 37.62 & 5.88 & 40.83 \\
\hline G. 86 XCB 58 & 33 & 60.73 & 23.98 & 3.48 & 10.93 & 39.47 & 7.13 & 39.67 \\
\hline G. 86 X(G. 75XSea) & 34 & 50.34 & 20.51 & 3.79 & 11.96 & 40.81 & 8.26 & 38.00 \\
\hline G. 86XAust12 & 35 & 51.68 & 20.70 & 3.40 & 12.75 & 40.08 & 8.53 & 39.83 \\
\hline G. 94 XG. 93 & 36 & 69.28 & 24.64 & 2.68 & 10.50 & 35.59 & 5.80 & 40.67 \\
\hline G. 94 XCB 58 & 37 & 87.32 & 32.70 & 3.53 & 10.46 & 37.42 & 6.24 & 39.33 \\
\hline G. $94 X(G .75 X S e a)$ & 38 & 94.92 & 39.66 & 3.79 & 11.06 & 41.69 & 7.92 & 39.50 \\
\hline G. 94XAus. 12 & 39 & 52.12 & 19.82 & 3.12 & 11.02 & 38.00 & 6.75 & 38.67 \\
\hline G. 93 XCB 58 & 40 & 58.50 & 22.64 & 2.85 & 10.43 & 38.67 & 6.57 & 35.83 \\
\hline G. 93X(G. 75XSea) & 41 & 90.89 & 37.58 & 3.45 & 11.56 & 41.38 & 8.17 & 38.50 \\
\hline G. 93XAus. 12 & 42 & 71.19 & 25.33 & 2.92 & 11.06 & 35.59 & 6.11 & 39.00 \\
\hline CB 58X(G. 75XSea) & 43 & 74.84 & 29.73 & 3.38 & 11.46 & 39.71 & 7.55 & 39.83 \\
\hline CB 58XAus. 12 & 44 & 52.94 & 20.55 & 3.49 & 10.69 & 38.84 & 6.78 & 40.17 \\
\hline (G. 75XSea)XAus. 12 & 45 & 59.98 & 22.23 & 3.11 & 10.17 & 37.09 & 5.99 & 38.50 \\
\hline TNB & 46 & 55.34 & 20.09 & 3.05 & 9.68 & 36.31 & 5.52 & 36.00 \\
\hline Kar. 2 & 47 & 46.76 & 15.19 & 2.74 & 10.24 & 32.49 & 4.93 & 37.67 \\
\hline G. 45 & 48 & 45.41 & 14.88 & 2.92 & 10.35 & 32.73 & 5.04 & 40.00 \\
\hline (G89XG86) & 49 & 65.23 & 24.87 & 3.48 & 10.52 & 38.15 & 6.50 & 39.50 \\
\hline G. 86 & 50 & 66.16 & 26.58 & 3.55 & 10.72 & 40.19 & 7.20 & 38.67 \\
\hline G. 94 & 51 & 63.52 & 26.31 & 3.92 & 12.70 & 41.41 & 8.98 & 39.33 \\
\hline G. 93 & 52 & 61.27 & 21.49 & 2.85 & 10.73 & 35.09 & 5.80 & 39.33 \\
\hline CB 58 & 53 & 47.32 & 16.56 & 2.97 & 10.16 & 34.99 & 5.47 & 39.83 \\
\hline G. 75XSea & 54 & 98.98 & 41.38 & 3.90 & 12.03 & 41.80 & 8.64 & 40.00 \\
\hline Aus. 12 & 55 & 58.70 & 21.18 & 3.22 & 10.53 & 36.12 & 5.96 & 36.83 \\
\hline LSD .01 & & 11.99 & 4.74 & 0.65 & 1.10 & 1.43 & 0.77 & 1.71 \\
\hline
\end{tabular}

Also, the parent Giza $94\left(\mathrm{P}_{6}\right)$ recorded the highest values for boll weight, seed index and lint index with the mean values of 3.92, 12.7 and $8.98 \mathrm{~g}$. respectively. While the parents Karsheneski ${ }_{2}\left(P_{2}\right)$ and Giza $45\left(P_{3}\right)$ gave low mean performance in most characters. For crosses the results cleared that, the crosses Giza 45 x CB 58, Giza 45 x (Giza $75 \times$ Sea), Giza 94 x (Giza 75 x Sea) and Giza 93 x (Giza 75 x Sea) were the highest crosses for seed cotton yield / plant, lint yield / plant, 
boll weight and lint percentage. For seed index and lint index results cleared that the crosses Giza 86 × (Giza 75 x Sea) and Giza $86 \times$ Aus. $_{12}$ were the highest crosses for seed index and lint index. For hallo length character the crosses TNB x Giza 94, Kar.2 x Giza 93, Giza 86 x Giza 93, Giza 94 x Giza 93 and CB 58 x Aus.12 were the highest crosses. These results were almost similar to that of Seyam et al., 1984 and Abdel-Hafez et al. (2016).

\section{General combining ability (GCA)}

Results of general combining ability effects of parental genotypes were obtained for studied characters (Table 3 ). The results exhibited that positive general combining ability effect was found for most studied characters, the comparison of GCA effect of parent exhibited the parental genotypes Giza 75 x Sea followed by Giza 94 were observed to be a good combiner for all studied characters in the same trend Giza 86 showed a good combiner for most yield contributed characters. On the other hand the parental Giza 93 showed a good combiner for seed cotton yield with

the best value for hallo length. However, the Russian genotype, Kar.2, followed by

Table 3: General combining ability effects of parental genotypes for all studied characters

\begin{tabular}{|c|c|c|c|c|c|c|c|}
\hline Parents & SCY/Plant & LCY/Plant & BW, g & SI,g & LP, \% & LI,g & $\begin{array}{c}\text { H } \\
\text { L,mm }\end{array}$ \\
\hline TNB & -2.859 & $-1.951^{\star}$ & 0.019 & $-0.322^{\star}$ & $-1.303^{\star}$ & $-0.564^{\star}$ & $-0.564^{\star}$ \\
\hline Kar. 2 & $-7.256^{\star}$ & $-4.196^{\star}$ & $-0.253^{\star}$ & $-0.454^{\star}$ & $-2.530^{\star}$ & $-0.923^{\star}$ & 0.006 \\
\hline G.45 & $-4.427^{\star}$ & $-1.961^{\star}$ & $-0.146^{\star}$ & $-0.366^{\star}$ & $-0.855^{\star}$ & $-0.457^{\star}$ & 0.019 \\
\hline (G89XG86) & 0.461 & 0.447 & -0.010 & -0.096 & $0.667^{\star}$ & 0.090 & -0.175 \\
\hline G.86 & 0.516 & $1.183^{\star}$ & $0.129^{\star}$ & $0.317^{\star}$ & $1.851^{\star}$ & $0.698^{\star}$ & 0.214 \\
\hline G. 94 & $2.563^{\star}$ & $1.595^{\star}$ & $0.175^{\star}$ & $0.555^{\star}$ & $1.086^{\star}$ & $0.649^{\star}$ & $0.339^{\star}$ \\
\hline G.93 & $4.105^{\star}$ & $0.973^{\star}$ & $-0.148^{\star}$ & 0.012 & $-0.727^{\star}$ & $-0.204^{\star}$ & $0.367^{\star}$ \\
\hline CB 58 & -0.520 & -0.242 & -0.012 & -0.141 & -0.088 & $-0.124^{\star}$ & 0.089 \\
\hline G75XSea & $11.592^{\star}$ & $6.045^{\star}$ & $0.274^{\star}$ & $0.455^{\star}$ & $2.123^{\star}$ & $0.893^{\star}$ & $0.339^{\star}$ \\
\hline Aus. 12 & -4.176 & $-1.862^{\star}$ & -0.027 & 0.041 & $-0.224^{\star}$ & -0.058 & $-0.633^{\star}$ \\
\hline
\end{tabular}

* Significant probability at 0.05

the Egyptian variety Giza 45 was observed to be a poor combiner for all yield Characters. Thus, the breeder may utilize the good general combiner such as the genotypes Giza 75 x Sea, Giza 94 and Giza 86 in specific breeding programs for improving most yield characters in our cotton. Theses results were in harmony with similar results reported by Amein et al. (2013) and Abdel- Hafez et al. (2016). 


\section{Specific combing ability (SCA)}

Specific combing ability effects (SCA) are given in Table 4. Significant positive SCA effects were obtained for some crosses indicating the presence of a considerable non-allelic gene effect. On the other hand, the significant negative estimates of SCA revealed the presence of undesirable types of epistasis in these combinations. The positive specific combining ability effects were found for variable traits in (16 crosses) Giza 45 x CB 58 for seed cotton yield/plant, lint yield/plant, boll weight, seed index, lint percentage and lint index. Also, the crosses Kar..2 x Giza 94, Giza 45 x Giza 86, Giza 94 x (Giza 75 x Sea) and Giza 93 x (Giza 75 x Sea) had positive specific combining ability effects for seed cotton yield / plant, lint yield / plant and lint percentage.

Table 4: Specific combining ability for all studied characters

\begin{tabular}{|c|c|c|c|c|c|c|c|}
\hline Genotypes & SCY/Plant & LCY/Plant & BW, g & SI, $\mathrm{g}$ & LP, $\%$ & LI, $\mathbf{g}$ & H L, mm \\
\hline TNBXKar. 2 & -1.37 & -0.440 & -0.246 & 0.029 & -0.360 & 0.008 & -0.451 \\
\hline TNBXG .45 & $-6.52^{\star}$ & $-3.240^{\star}$ & -0.073 & $0.641^{\star}$ & $-1.595^{\star}$ & -0.012 & $1.535^{\star}$ \\
\hline TNBX(G. 89XG. 86) & $-6.86^{\star}$ & -2.090 & 0.032 & 0.255 & 0.613 & 0.315 & 0.563 \\
\hline TNBXG. 86 & 1.59 & 0.960 & 0.086 & -0.182 & 0.399 & -0.032 & $1.174^{\star}$ \\
\hline TNBXG. 94 & $9.27^{\star}$ & 1.750 & $0.500^{\star}$ & $0.963^{\star}$ & $-2.366^{\star}$ & -0.171 & $1.716^{\star}$ \\
\hline TNBXG. 93 & $6.15^{\star}$ & 1.760 & $0.403^{\star}$ & 0.106 & -0.599 & -0.071 & $1.355^{\star}$ \\
\hline TNBxCB 58 & $-17.40^{\star}$ & $-6.080^{\star}$ & -0.194 & -0.324 & 0.588 & -0.028 & $-1.867^{\star}$ \\
\hline TNBxG. 75XSea & $-7.34^{*}$ & $-3.140^{\star}$ & -0.106 & -0.203 & -0.013 & -0.204 & 0.049 \\
\hline TNBXAus. 12 & $27.21^{*}$ & $10.270^{\star}$ & 0.005 & $-0.906^{\star}$ & 0.387 & $-0.404^{\star}$ & -0.645 \\
\hline Kar. 2XG. 45 & $-20.88^{\star}$ & $-7.330^{\star}$ & -0.085 & 0.030 & -0.454 & -0.043 & 0.299 \\
\hline Kar. 2X(G. 89XG. 86) & $22.48^{*}$ & $7.580^{*}$ & 0.157 & -0.146 & -0.564 & -0.223 & -0.006 \\
\hline Kar. 2XG. 86 & $14.14^{*}$ & $5.150^{\star}$ & -0.259 & -0.323 & -0.041 & -0.287 & -0.562 \\
\hline Kar. 2XG. 94) & $7.89^{\star}$ & $3.330^{*}$ & 0.189 & 0.372 & $0.931^{\star}$ & 0.361 & 0.480 \\
\hline Kar. 2XG. 93 & $10.78^{*}$ & $3.080^{\star}$ & 0.152 & -0.332 & $-0.792^{\star}$ & -0.335 & 2.619 \\
\hline Kar. 2XCB 58 & $7.54^{\star}$ & 1.900 & -0.125 & -0.178 & $-1.012^{\star}$ & -0.319 & 0.730 \\
\hline Kar. 2X(G. 75XSea) & $-24.45^{\star}$ & $-9.190^{\star}$ & 0.069 & -0.225 & $1.058^{\star}$ & 0.014 & -0.020 \\
\hline Kar. 2XAus. 12 & $-11.85^{\star}$ & $-3.500^{\star}$ & 0.084 & -0.490 & $1.014^{\star}$ & -0.028 & -0.715 \\
\hline G. 45X(G. 89XG. 86) & 3.89 & $2.960^{\star}$ & -0.284 & 0.185 & $2.742^{\star}$ & $0.851^{*}$ & $-2.354^{\star}$ \\
\hline G. 45XG. 86 & $8.80^{*}$ & $4.120^{*}$ & $0.377^{\star}$ & 0.029 & $1.378^{\star}$ & 0.347 & 0.258 \\
\hline G. 45XG. 94 & $-15.84^{\star}$ & $-6.840^{\star}$ & -0.299 & $-1.030^{\star}$ & $-1.203^{\star}$ & $-0.985^{\star}$ & 0.299 \\
\hline G. 45XG. 93 & $-14.93^{\star}$ & $-4.340^{\star}$ & $0.345^{\star}$ & 0.100 & $2.623^{\star}$ & $0.729^{\star}$ & $-2.895^{\star}$ \\
\hline G. 45XCB 58 & $36.90^{*}$ & $15.670^{\star}$ & $0.348^{*}$ & $0.784^{\star}$ & $2.643^{\star}$ & $1.181^{*}$ & -0.284 \\
\hline G. 45X(G. 75XSea) & $24.37^{*}$ & $7.810^{*}$ & $-0.388^{\star}$ & -1.386 & $-1.073^{\star}$ & $-1.222^{\star}$ & 0.633 \\
\hline G. 45XAus12 & 2.52 & 1.340 & 0.067 & -0.475 & $0.943^{\star}$ & -0.048 & 0.271 \\
\hline (G. 89XG. 86)XG86 & 4.94 & 1.960 & $-0.481^{*}$ & -0.018 & -0.381 & -0.113 & 0.619 \\
\hline (G. 89XG. 86)X G. 94 & $-8.47^{\star}$ & $-2.780^{\star}$ & $-0.517^{\star}$ & -0.519 & $0.714^{\star}$ & -0.148 & $-1.173^{\star}$ \\
\hline (G. 89XG. 86)XG. 93 & $-6.17^{*}$ & -2.170 & 0.196 & 0.330 & 0.044 & 0.215 & $0.966^{\star}$ \\
\hline (G. 89XG. 86)XCB 58 & -2.19 & -1.380 & 0.099 & $-0.830^{\star}$ & $-0.909^{\star}$ & $-0.736^{\star}$ & 0.244 \\
\hline (G. 89XG. 86)XG. 75XSea & $-10.78^{\star}$ & $-5.050^{\star}$ & 0.180 & 0.044 & $-1.140^{*}$ & -0.322 & 0.327 \\
\hline (G. 89XG. 86)xAus12 & 1.39 & 0.770 & 0.048 & 0.302 & 0.143 & 0.226 & $-1.201^{\star}$ \\
\hline G. $86 X$ G. 94 & $-11.52^{\star}$ & $-4.010^{\star}$ & -0.066 & -0.319 & $0.837^{\star}$ & 0.064 & -2.229 \\
\hline G. 86XG. 93 & $14.56^{\star}$ & $5.020^{\star}$ & -0.197 & $-1.103^{\star}$ & $-0.947^{\star}$ & $-0.966^{\star}$ & $1.410^{*}$ \\
\hline G. 86XCB 58 & -2.68 & -0.830 & 0.144 & 0.237 & 0.267 & 0.210 & 0.521 \\
\hline G. 86X(G. 75XSea) & $-25.19^{\star}$ & $-10.560^{\star}$ & 0.171 & $0.674^{\star}$ & -0.607 & 0.324 & $-1.395^{\star}$ \\
\hline G. 86XAust12 & $-8.08^{\star}$ & $-2.500^{\star}$ & 0.079 & $1.875^{\star}$ & $1.010^{\star}$ & $1.541^{*}$ & $1.410^{\star}$ \\
\hline G. 94XG. 93 & 0.81 & -1.800 & $-0.559^{\star}$ & $-0.581^{\star}$ & -2.215 & $-0.997^{\star}$ & $1.119^{\star}$ \\
\hline G. 94XCB 58 & $21.86^{*}$ & $7.470^{\star}$ & 0.151 & -0.474 & $-1.018^{\star}$ & $-0.635^{\star}$ & 0.063 \\
\hline G. 94X(G. 75XSea) & $17.35^{\star}$ & $8.180^{\star}$ & 0.125 & -0.471 & $1.035^{\star}$ & 0.033 & -0.020 \\
\hline G. 94XAus. 12 & $-9.68^{*}$ & $-3.790^{\star}$ & -0.240 & -0.093 & -0.308 & -0.187 & 0.119 \\
\hline G. 93 XCB 58 & $-8.50^{\star}$ & -1.970 & -0.203 & 0.039 & $2.045^{\star}$ & $0.549^{\star}$ & $-3.465^{\star}$ \\
\hline G. 93X(G. 75XSea) & $11.78^{*}$ & $6.720^{*}$ & 0.112 & $0.579^{\star}$ & $2.542^{\star}$ & 1.129 & $-1.048^{\star}$ \\
\hline G. 93XAus. 12 & $7.85^{\star}$ & $2.350^{\star}$ & -0.117 & 0.493 & $-0.905^{\star}$ & 0.020 & 0.424 \\
\hline CB 58X(G. 75XSea) & 0.36 & 0.090 & -0.095 & $0.632^{\star}$ & 0.232 & $0.432^{\star}$ & 0.563 \\
\hline CB 58XAus. 12 & -5.78 & -1.220 & 0.313 & 0.270 & $1.712^{\star}$ & $0.616^{\star}$ & $1.869^{*}$ \\
\hline (G. 75XSea)XAus. 12 & $-10.85^{\star}$ & $-5.800^{\star}$ & $-0.349^{\star}$ & $-0.843^{\star}$ & $-2.252^{\star}$ & $-1.190^{\star}$ & -0.048 \\
\hline
\end{tabular}

${ }^{\star}$ Significant probability at 0.05 
Also, the cross TNB $\times$ Australian $_{12}$ showed positive specific combining ability effects for seed cotton yield / plant, lint yield / plant and hallo length. Concerning seed cotton yield / plant and hallo length the crosses TNB x Giza 94 and TNB x Giza 93 exhibited positive specific combining ability effects. Also, the crosses Giza 86 x Aus.12 and CB $58 \times$ Aus. $_{12}$ had positive specific combining ability effects for lint percentage and hallo length. The results reported by Khan et al. (2011), Amein et al. (2013) and Abdel-Hafez et al. (2016) agreed with the present one.

It's important to note that the most of combinations having significant SCA effect were between genetically diverse parents as stated by El- Mansy et al, (2014). Most combinations which had good specific combining ability were having one or two parents of either good $x$ good or good $x$ poor general combiner.

\section{Better parent (BP.)}

The amount of heterosis versus the better parent (BP.) is presented in Table (5). The results exhibited that the crosses Giza $45 x$ CB 58 was superior and positive heterosis for most characters, seed cotton yield, lint yield, lint percentage and lint index, with the mean heterosis values of $101.52 \%, 125.45 \%, 11.86 \%$ and $27.13 \%$ respectively. On the other hand, the crosses TNB x Aus.12, Kar.2 x CB 58 and Giza 94 x CB 58 were the best and showed the highest positive heterosis values for seed cotton yield / plant and lint yield / plant of (42.39\%, $43.22 \%),(33.49 \%, 28.85 \%)$ and $(37.47,24.31 \%)$ respectively. In the same time, for seed index in the cross Giza $86 x$ Aus. ${ }_{12}$ was the best cross with highest positive heterosis value of 18.97 $\%$. Also, the crosses Kar.2 x Giza 45, Giza 45 x Giza 94 and Giza 45 x Aus. ${ }_{12}$ exhibited the best and highest positive heterosis values for lint percentage with a mean heterosis values of $3.41 \%, 4.85 \%, 9.67 \%$ and $3.27 \%$ respectively. Also, high positive heterosis values for lint index of $18.47 \%, 13.21 \%$ and $13.81 \%$ were obtained for the crosses Giza 86 x Aus. ${ }_{12}$, Giza 93 x CB 58 and CB 58 X Aus. 12 respectively. The superior positive heterosis for hallo length of $6.35 \%, 3.81 \%, 3.39$ $\%$ and $10.04 \%$ for Kar. $x$ x Giza 93, Giza 86 x Giza 93, Giza 94 x Giza 93 and Giza 93 x CB 58 respectively were found. The results cleared that no one cross from all crosses was superior and showed high positive heterosis for all the studied characters. These results were harmony with Sorour et al., 2013 and Abou El- Yazied et al., 2009. 
Table 5: Heterosis relative to better parent (BP.) for all studied characters

\begin{tabular}{|c|c|c|c|c|c|c|c|}
\hline Genotypes & SCY/Plant & LCY/Plant & BW, g & SI, g & LP, \% & LI, g & H L, mm \\
\hline TNBXKar. 2 & -6.171 & -13.985 & -10.273 & -4.590 & $-8.422^{\star \star}$ & $-11.775^{\star}$ & 0.442 \\
\hline TNBXG .45 & -10.359 & -16.780 & -1.093 & 1.192 & $-7.210^{\star \star}$ & -3.684 & -0.417 \\
\hline TNBX(G. 89XG. 86) & -16.980 & $-18.430^{\star}$ & -6.418 & -1.615 & $-1.905^{\star \star}$ & -4.720 & -2.110 \\
\hline TNBXG. 86 & -5.290 & -9.467 & -2.726 & -3.608 & $-4.473^{\star \star}$ & $-10.417^{\star \star}$ & 2.586 \\
\hline TNBXG. 94 & 13.960 & -3.960 & -0.340 & $-7.793^{\star}$ & $-15.826^{\star \star}$ & $-30.252^{\star \star}$ & 2.542 \\
\hline TNBxG. 93 & $15.568^{\star}$ & 14.723 & 14.426 & -3.854 & $-4.116^{\star \star}$ & -5.055 & 1.695 \\
\hline TNBxCB 58 & $-22.963^{\star \star}$ & $-22.337^{\star}$ & -0.656 & -4.201 & 0.915 & 2.053 & $-8.368^{\star \star}$ \\
\hline TNBxG. 75XSea & $-34.529^{\star \star}$ & $-40.081^{\star \star}$ & $-12.810^{\star}$ & $-13.137^{\star \star}$ & $-8.482^{\star \star}$ & $-25.048^{\star \star}$ & $-3.333^{\star}$ \\
\hline TNBXAus. 12 & $42.391^{\star \star}$ & $43.221^{\star \star}$ & -0.104 & $-11.424^{\star \star}$ & -0.013 & $-10.682^{\star}$ & 0.453 \\
\hline Kar. 2XG. 45 & $-34.020^{\star \star}$ & $-32.649^{\star \star}$ & -6.393 & -5.992 & $3.412^{*}$ & -2.184 & -2.083 \\
\hline Kar. 2X(G. 89XG. 86) & $21.273^{\star \star}$ & 11.413 & -10.632 & -6.683 & $-8.205^{\star \star}$ & $-18.522^{\star \star}$ & -2.110 \\
\hline Kar. 2XG. 86 & 7.041 & -2.151 & $-20.113^{\star \star}$ & -6.159 & $-8.620^{\star \star}$ & $-18.935^{\star \star}$ & -0.431 \\
\hline Kar. 2XG. 94) & 4.881 & -6.456 & $-15.208^{\star}$ & $-13.487^{\star \star}$ & $-10.828^{\star \star}$ & $-28.322^{\star \star}$ & 0.848 \\
\hline Kar. 2XG. 93 & $15.948^{\star}$ & 10.358 & 3.972 & $-9.168^{\star}$ & $-4.832^{\star \star}$ & $-15.795^{\star \star}$ & $6.356^{\star \star}$ \\
\hline Kar. 2XCB 58 & $33.496^{\star \star}$ & $28.855^{\star \star}$ & -4.933 & -4.851 & $-3.370^{\star \star}$ & -8.842 & -0.418 \\
\hline Kar. 2X(G. 75XSea) & $-56.252^{\star \star}$ & $-60.128^{\star \star}$ & $-15.286^{\star}$ & $-14.413^{\star \star}$ & $-8.857^{\star \star}$ & $-26.669^{\star \star}$ & -2.083 \\
\hline Kar. 2XAus. 12 & $-31.629^{\star \star}$ & $-32.387^{\star \star}$ & -6.114 & $-8.734^{\star}$ & -1.163 & $-10.403^{\star}$ & -0.443 \\
\hline G. 45X(G. 89XG. 86) & -2.900 & 1.816 & $-20.211^{\star \star}$ & -2.692 & $4.850^{\star \star}$ & 5.182 & $-9.167^{\star \star}$ \\
\hline G. 45XG. 86 & 3.237 & 2.370 & 0.846 & -2.053 & -0.923 & -3.657 & -1.667 \\
\hline G. 45XG. 94 & $-28.037^{\star \star}$ & $-36.628^{\star \star}$ & -24.894 & $-23.825^{\star \star}$ & $-11.939^{\star \star}$ & $-38.122^{\star \star}$ & -1.250 \\
\hline G. 45XG. 93 & $-21.400^{\star \star}$ & -13.746 & 11.872 & -4.320 & $9.674^{\star \star}$ & $10.569^{\star}$ & $-9.167^{\star \star}$ \\
\hline G. 45XCB 58 & $101.520^{\star \star}$ & $125.453^{\star \star}$ & 14.574 & 4.316 & $11.862^{\star \star}$ & $27.134^{\star \star}$ & $-3.333^{\star}$ \\
\hline G. 45X(G. 75XSea) & -4.067 & $-13.630^{\star \star}$ & $-24.253^{\star \star}$ & $-23.337^{\star \star}$ & $-9.950^{\star \star}$ & $-35.585^{\star \star}$ & -0.417 \\
\hline G. 45XAus 12 & -2.328 & 0.999 & -3.316 & -7.753 & $3.276^{*}$ & -2.908 & $-3.750^{\star}$ \\
\hline (G. 89XG. 86)XG86 & 4.799 & 3.292 & $-19.549^{\star \star}$ & 0.031 & $-1.512^{\star}$ & -2.454 & 0.000 \\
\hline (G. 89XG. 86)X G. 94 & -11.128 & -12.057 & $-27.018^{\star \star}$ & $-17.685^{\star \star}$ & $-3.632^{\star \star}$ & $-22.717^{\star \star}$ & $-4.219^{\star}$ \\
\hline (G. 89XG. 86)XG. 93 & -5.238 & -7.037 & -6.514 & 0.341 & $-1.887^{\star \star}$ & -0.718 & 1.266 \\
\hline (G. 89XG. 86)XCB 58 & -6.222 & -8.746 & -5.364 & $-10.199^{\star}$ & -2.709 & $-14.110^{\star \star}$ & -2.092 \\
\hline (G. 89XG. 86)XG.75XSea & $-34.647^{\star \star}$ & $-38.893^{\star}$ & -6.234 & $-9.202^{\star \star}$ & $-6.465^{\star \star}$ & $-18.834^{\star \star}$ & -1.667 \\
\hline (G. 89XG. 86)xAus12 & 4.060 & -6.602 & -7.280 & 2.184 & -0.306 & 1.693 & $-6.751^{\star \star}$ \\
\hline G. $86 X$ G. 94 & $-16.904^{*}$ & $-14.828^{\star}$ & -11.980 & $-12.857^{\star \star}$ & -0.477 & -13.586 & $-5.508^{\star \star}$ \\
\hline G. 86XG. 93 & $24.846^{\star \star}$ & $16.821^{\star \star}$ & $-15.414^{\star}$ & $-9.168^{\star}$ & $-6.389^{\star \star}$ & $-18.380^{\star \star}$ & $3.814^{\star}$ \\
\hline G. 86 XCB 58 & -8.200 & -9.781 & -1.974 & 1.990 & -1.777 & -0.926 & -0.418 \\
\hline G. 86X(G. 75XSea) & $-49.145^{\star \star}$ & $-50.433^{\star \star}$ & -2.903 & -0.527 & -2.357 & -4.323 & $-5.000^{\star \star}$ \\
\hline G. 86XAust12 & -11.968 & $-22.151^{\star \star}$ & -4.229 & $18.973^{\star \star}$ & -0.267 & $18.472^{\star \star}$ & 3.017 \\
\hline G. 94XG. 93 & 9.066 & -6.330 & $-31.606^{\star \star}$ & $-17.318^{\star \star}$ & -14.071 & $-35.449^{\star \star}$ & $3.390^{\star \star}$ \\
\hline G. 94 XCB 58 & $37.471^{\star \star}$ & $24.317^{\star \star}$ & -10.025 & $-17.685^{\star \star}$ & -9.637 & $-30.512^{\star \star}$ & -1.255 \\
\hline G. 94X(G. 75XSea) & -4.101 & -4.157 & -3.398 & $-12.962^{\star \star}$ & -0.260 & $-11.767^{\star \star}$ & -1.250 \\
\hline G. 94XAus. 12 & -11.216 & $-24.672^{\star \star}$ & $-20.391^{\star \star}$ & $-13.251^{\star \star}$ & -8.252 & $-24.796^{\star \star}$ & -1.695 \\
\hline G. 93 XCB 58 & -4.521 & 5.303 & -4.036 & -2.797 & 10.215 & $13.211^{\star \star}$ & $-10.042^{\star \star}$ \\
\hline G. 93X(G. 75XSea) & -8.167 & $-9.188^{\star}$ & -11.529 & -3.853 & -0.993 & -5.442 & $-3.750^{\star}$ \\
\hline G. 93XAus. 12 & $16.193^{\star \star}$ & $17.848^{\star}$ & -9.119 & 3.138 & -1.485 & 2.461 & -0.847 \\
\hline CB 58X(G. 75XSea) & $-24.383^{\star \star}$ & $-28.143^{\star \star}$ & $-13.322^{\star}$ & -4.685 & -4.989 & $-12.582^{\star}$ & -0.417 \\
\hline CB 58XAus. 12 & -9.810 & -2.974 & 8.497 & 1.456 & 7.530 & $13.814^{\star \star}$ & 0.837 \\
\hline (G. 75XSea)XAus. 12 & $-39.404^{\star \star}$ & $-46.276^{\star \star}$ & -3.213 & $-15.438^{\star \star}$ & -11.258 & $-30.606^{\star \star}$ & $-3.750^{*}$ \\
\hline
\end{tabular}

${ }^{\star *}$ and ${ }^{*}$ significant at 0.05 and 0.01 levels of probability respectively.

\section{Principal component}

Principal component analysis (PCA) reflects the important of the largest contributor to the total variation at each ax for differentiation Sharma, (1998). Principal component analysis seemed to elucidate patterns of variation in agronomic attributes which are of economic importance and obtain entail factor solution using Eigen values. These values could measure the explained variance associated with each variable Hair et al., (1987). The first three principal components (PCS) whose Eigen values were greater than one were significant and accounted $90.3 \%$ of total variation of all characters (Table 6). The first PC explained about $54.2 \%$ of the all total variation with the highest Eigen value of 3.7935 , the second explain $21.5 \%$ with Eigen value of 1.5066, the third explained $14.6 \%$ of the total variation with Eigen value of 1.0227 . 
According to (Chahal and Gosal, 2002) characters with largest absolute values closer to unity within the first principal component influence the clustering more than those with lower absolute values closer to zero.

Thus, it is possible to include the corresponding amount of variance in a two dimensional plot of the components. Each genotype is plotted at it is principal component score on each axes (Brown, 1991). Each character was an important source of variation in at least one PC axes, because each of PC axes was given equal weight in the multivariate analysis. Thus each of character was contributed to the information which used to group genotypes; however some characters may have greater importance in determine plant phenotypes than others. Lint index followed by lint percentage, boll weight, lint yield as well as seed index were a primary source of variation with the largest coefficient in the first PC axes respectively. Thus, the first PC ax was correlated with yield and weighted characters with positive loadings. While the second PC ax was principally affected by seed cotton yield with hallo length. The other rest axes deals with hallo length which showed negative loading. In this connection Abdel- Salam et al, (2010) and El- Mansy et al, (2014).

The present study confirmed that cotton genotypes showed wide amount of variation for studied characters and it also suggests ample opportunities for genetic improvement of cotton genotypes. Each component score is a linear combination of the characters, similar to an index, such that the maximal amount of variance is shown in the first and second PC, etc. The two dimensional distance between genotypes might reflect a summary of differences based on all characters measured to the extent that the first two PC axes are effective in capturing the combined variance of most characters (Fig. 1). Therefore, the first two PC axes were used to plotting the studied parental genotypes and $F_{1}$ hybrids. In this connection Hair et al., (1987), Sharma, (1998), You et al., (1998), Abd El- Sayyed et al., (2000) and El-Lawendy et al., (2008).

On the basis of the relative contribution of the studied yield and fiber characters, the 10 parental genotypes and $45 F_{1}$ crosses combinations were grouped into varied genotypes (Figure 1). The parental genotypes were separated into varied groups. The parental genotype Giza 75 x Sea formed unique group, however the other two varieties Giza 86 and Giza 94 clustered in one group and nearly related with Giza $75 \times$ Sea group. Such genotypes characterized as a good combiner for most yield characters. On the other side Giza 93 located in group with some $F_{1}$ combinations. This parent was a good combiner for hallo length character. 
Table 6: Principal components (PC) analysis of characters associated with nine cotton genotypes showing Eigen values and proportion variation associated with the five $\mathrm{PC}$ axes and Eigen vector of characters

\begin{tabular}{|c|c|c|c|c|c|}
\hline Variable & PC1 & PC2 & PC3 & PC4 & PC5 \\
\hline Eigen value & 3.7935 & 1.5066 & 1.0227 & 0.3839 & 0.2886 \\
\hline Proportion & 0.542 & 0.215 & 0.146 & 0.055 & 0.041 \\
\hline Cumulative & 54.2 & 75.7 & 90.3 & 95.8 & 99.9 \\
\hline \multicolumn{5}{|c|}{ Eigen vector } \\
\hline SCY/Plant & 0.638 & 0.723 & 0.229 & 0.013 & 0.133 \\
\hline LCY/Plant & 0.771 & 0.574 & 0.267 & 0.04 & 0.042 \\
\hline BW, g & 0.799 & -0.146 & -0.208 & -0.546 & 0.009 \\
\hline Sl, g & 0.756 & -0.393 & -0.356 & 0.196 & 0.33 \\
\hline LP, \% & 0.848 & -0.288 & 0.275 & 0.096 & -0.336 \\
\hline Ll, g & 0.911 & -0.375 & 0.002 & 0.161 & -0.054 \\
\hline H L, mm & 0.188 & 0.506 & -0.809 & 0.104 & -0.21 \\
\hline
\end{tabular}

Five Parental genotypes TNB, Kar.2, Giza 45, Aus. 12 and CB 58 were grouped in the same group and characterized as poorer parent for GCA for yield and fiber characters these parents were isolated by the second PC axes. On the basis of SCA most combination having significant SCA effect was between genetically diverse parents. On the other side most $F_{1}$ combinations which formed wide distance groups were between parents in different clusters.

It is evident to no that crossing of distantly related parents may give best hybrids which surpassed their parents in most characters and should produce higher variances for most characters in segregating generations rather than crossing between closed related parents which agree with Suinaga et al., (2005) and El- Mansy et al, (2014).

From a plant breeding principal component analysis is useful in identifying and the most influential characters affecting genetic variation of plant population. The loading of morphological and agronomic characters of an individual genotype indicate the magnitude of genetic variation. 


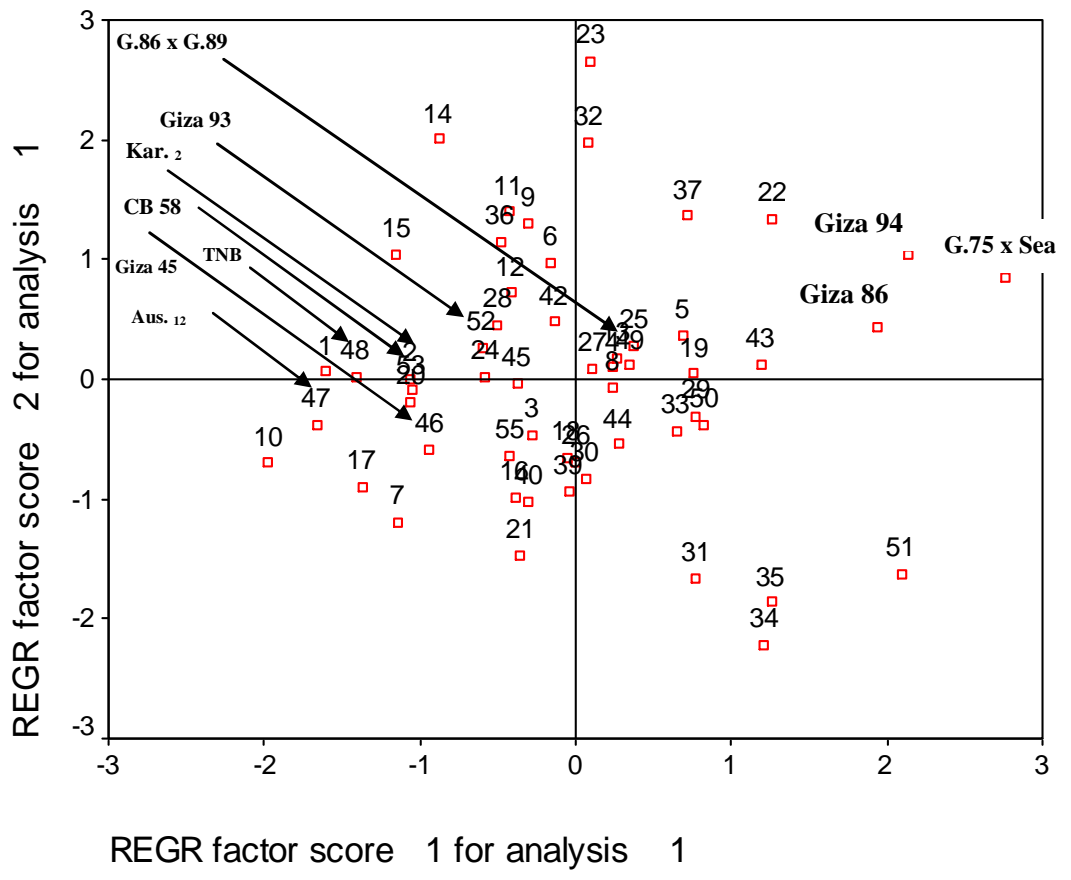

Figure 1: Representation of 55 cotton genotypes of the first two PC axes of principal component analysis

\section{REFERENCE}

Abdel-Hafez,A.G.,A.E.I. Darwesh, F.A. Sorour and Aml S.Abdel-Hafez, 2016. Performance of some genotypes of cotton under conventional and late planting dates. J. Agric. Res. Kafr ElSheikh, Univ., A. Plant production, Egypt 42 (3):336-352.

Abd EL- Sayyed, S. M.; A. R. Abo- Arab and Y. M. El- Mansy (2000). Genetic divergence among cotton genotypes J Agric. Sci Mansoura Univ.25 (11): 6643- 6657.

Abdel Salam, M.E., Y.M.El-Mansy and Rokia, M.Hassan (2010). The relative importance of characters affecting genetic divergence in cotton. J. Agric. Res. Kafr El-Sheikh, Univ., Egypt 36 (1):44-6. 
Abou EL-Yazzied, M. A.; H. M. Hamoud and M. A. Abd El- Gelil (2009). Hybrid performance as related to parental diversity in cotton $E$ gypt. J APPI. Sci 24(1) 240- 253.

Abou El-Yazzied, M. A.; W. M. B. Yehia; and H. A. El-Hoseiny, 2014. Genetic behavior of $F 1$ and $F 2$ for some economic traits in cotton crosses. Egypt J. Agric. Res.,92(1): 25-39.

Ahuja S.L., O. P. Tuteja, 2000. Variability and association analysis for chemical components imparting resistance in $G$. hirsutum $L$. cotton . J. Cotton Res. Dev, 14(10), 19-22.

Amein M.M.M., M.I. Masri, A.M.R. Abd El-Bary and S.S. Attia, 2013. Combinig ability and heterosis for yield and fiber quality traits in cotton (Gossypium barbadense L.). Egypt. J. plant breed. 17 (5), 129-141.

Chahal, G.S. and S. S. Gosal (2002) Principal and procedures of plant breeding. Biot. Conventional approaches, New Delhi.

El- Lawendey M. M.; Y. M., El- Mansy.and Y. A. Soliman (2008) Multivariate analysis of some economic characters in cotton varieties. Minufiya J. Agric.Res. Vol 33 (4): 55- 72.

El-Mansy, Y. M. (2005). Using genetic components for predicating new recombination in some cotton crosses (Gossypium barabdense L.) Ph.D. Thesis, Fac. of Agric. Mansoura Univ. Egypt.

El- Mansy, Y. M. (2009). Cluster analysis with selection index for improvement som economic characters in some cotton genotypes. 1st Nile Delta conf. Fac. of Agric. Minufiya Univ..

El- Mansy, Y. M.; M. M. El- Lawendey and M. E. Abd El- Salam (2008) inferior lint quality as a result of off type cotton plants existence in some Egyptian cotton varieties. J. Ageic. Res. Kafr El- Sheikh Univ.34 (1): 43- 53.

El- Mansy, Y. M.; M. E. Abd El- Salam and B. M. Ramdan (2014) Multivariate analysis of genetic divergence and combining ability in G. Barbadense L.. J. Ageic. Res. Kafr El- Sheikh Univ. 40 (1): 85- 103.

Gooda, B.M.R. (2007) Improvement of some economic characters in crosses of Egyptian cotton, Ph. D. Thesis. Fac. Agric. Kafr ElSheikh Univ. Egypt.

Griffing, B. (1956). Concepts of general and specific combining ability in relation to diallel crossing systems. Anst. J. Biol. Sci. 9.

Hair, J. F.; Jr. R. E. Anderson and R. L. Tatham (1987) Multivariate data analysis with Reading. Mac Millan Pub. Co. New York .

Khan T. M. , J. Farooq, A. Mahmood, R. N. Abbas, W. Nazeer , A. Farooq, Z. Hasnain, M. N. Akhtar, 2011. Exploring influential plant traits for enhancing upland cotton yield under salt stress. Front. Agric. China 2011, 5(4): 443-449.

Patil, S. A.; P. M. Salimath, M. B. Chtti, F. B. Patil and C. R. Konda (1999) Genetic divergence and heterosis in cotton. Crop Res. Hisar 18(2): 226-229.

Sandhu, B.S. and M. S. Boparai (1997) Genetic divergence in G.arboreum L. Indian J. Gent \& Plant Breed. 57(4): 461-465. 
Seyam, S.M.; A.A. aAbo El-Zahab, F.M. El-Rayes and H.N. El-Rassas (1984). Factor analysis of yield in Egyptian cotton. Agric. Res. Rev., 62(6): 33-40.

Sharma, J. R. (1998). Statistical and Biometrical techniques in plant breeding. New Age. Int. Ltd, Puna, India.

Snedecor, G.W. and W.G. Cochran, 1982. Statistical Methods, lowa state., Univ. Press., USA.

Sorour, F. A.; M. S., Abdel- Aty.; W. M. B Yehia and H. M. K. Kotb (2013) Hetrosis and combining ability in some cotton crosses in two different environments 1- yield and yield combonents. J. plant production, Mansoura Univ., vol. 4 (11): 1707 -1723.

SPSS (1995). SPSS computer user's guide, USA.

Suinaga, F.A.; E. C. Freire and L.E.P. Rangel (2005) Multivariate analysis of genetic divergence in cotton. This research was sponsored by FACUAL.

Tuteja O.P., P. Luthra and S. Kumar, 2003. Combining ability analysis in Upland cotton (Gossypium hirsutum) for yield and its components . Indian Agri. Sci. 73 (12), 671-675.

You, J., J.L. Liu and J.Z. Sun (1998). Analysis of heterosis and its components in interspecific crosses Acta.Agron Sinica 24(6): 834-839.

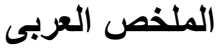

القدره على التألف وعلاقتها بالتباعد الوراثى فى أقطان الباربادينس

شاكر عبد العزيز شاكر - أشرف إبراهيم إسماعيل درويش - محمد عزت عبد السلام البرام معه بحوث القطن - مركز البحوث الزراعية ــ الجيزة ـ مصر

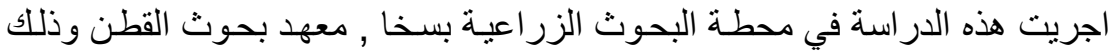

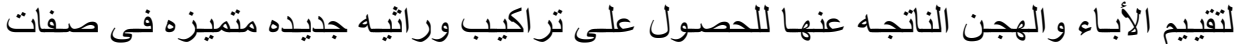

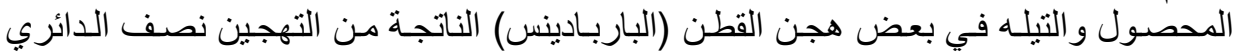
بين عشرة اباء وتقدير بعض المقاييس الور اثيه منل القدره العامه و الخاصنه (half-diallel ) على التألف واستخدام تكنيك التحليل المتعدد Multivariate analysis باستعمال المكونـات الأساسيه Principal components analysis لدار اسة الأهميه النسبيه للصفات المختلفه

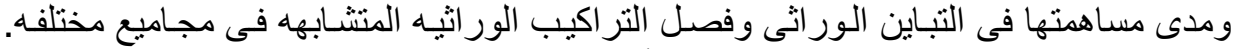

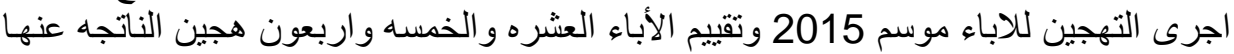

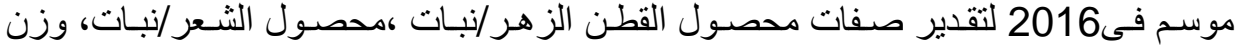

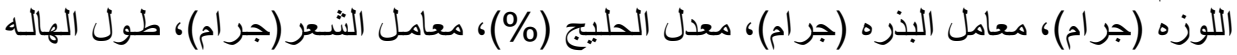
(ملليمنر).

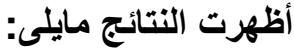
وجود اختلافـات عاليـة المعنويـه للتر اكيب الور اثيـه و القدره العامـه و الخاصـه للتـألف لكل 
Giza 94, Giza 75 x Sea النسبه لتقدير القدره الأعامـه على التألف كان الأبوين

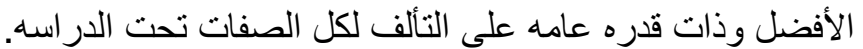
أظهرت تقدير ات القدره الخاصه على على التألف أن الهجن

Giza 45 x CB 58, TNB x Aus. 12, Kar. 2 x Giza 94, Giza 94 x (Giza 75 × Sea), Giza 93 × (G.75 x sea).

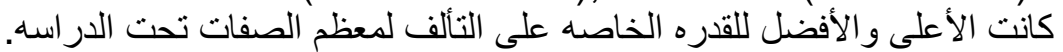
أظهرت النتائج بالنسبه لتقدير قوة الهجين مقارنة بأفضل الأباء أن الهجن Giza 45 x CB 58, TNB x Aus. 12, Kar. 2 x CB 58, Giza 94 x Giza 93, Kar.2 x Giza 45, Giza 45 x Giza.93, Giza 45 x Aus.12, Kar. 2 x Giza 93, Giza 93 x CB 58. تفوقت و أعطت قوة هجين موجبه لمعظم الصفات تحت الدار اسه مقارنة بباقي الهجن.

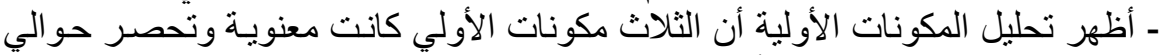

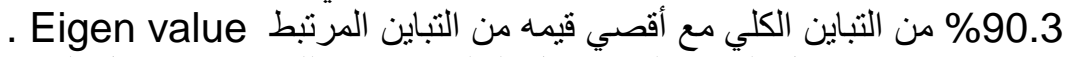

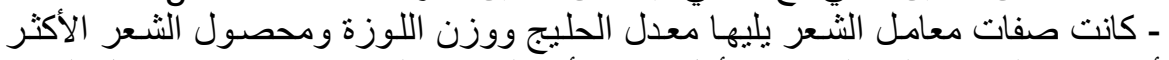

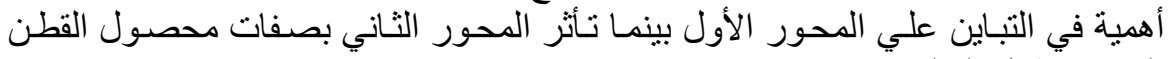

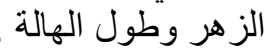

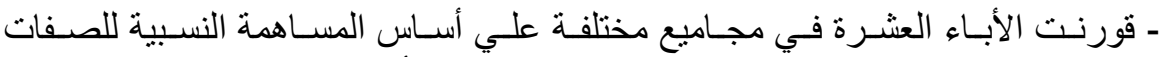

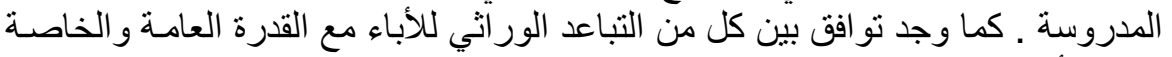

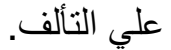

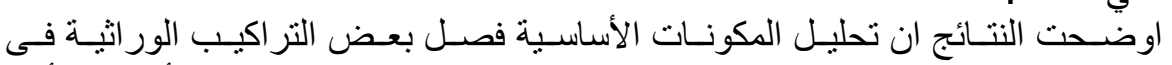

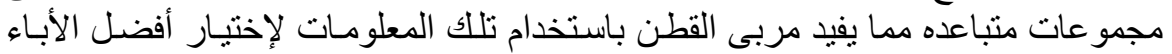
و الهجن لتحسين الصفات المختلفة في الأجيال الإنعز الية . 